\title{
SELF-HEATING BEHAVIOUR OF LOW MOISTURE CONTENT PARTICLES - MODELLING THE BASKET-HEATING OF SOLID PARTICLES AND SOME ASPECTS OF THE CROSS OVER BEHAVIOUR USING MILK POWDER AS AN EXAMPLE
}

\author{
XIAO DONG CHEN ${ }^{1}$
}

(Received 29 November, 1998; revised 12 January, 1999)

\begin{abstract}
Self-heating in packed particulate that is exothermically reactive is a major cause of fire and explosion in the powder industry. This study is focused on part of the Auckland development of a mathematical model dealing with this hazardous process in industry using milk powder as an example. Milk powder is a primary powdered food product around the world.

An update of the detailed mathematical model is given here, and predictions are made using the model to simulate the basket-heating behaviour of a milk powder in the laboratory (so the model can thus be validated). Basket heating in an oven is a standard laboratory technique for measuring the exothermic reactivity of a solid material.

After a favorable comparison with the laboratory results, several aspects of basket-heating were investigated with a view to further improving the technique. Firstly, the model was used to explore the effect of elevated ambient humidity and initial sample water content upon the heating process in the basket. Secondly, the model was used to explore the cross over phenomenon which is related to a novel procedure for measuring activation energy and exothermicity (that is, the Crossing-Point-Temperature (CPT) method, which is a new version of the basket heating technique). The predictions together with the experimental evidence show that the reaction kinetics obtained using the Heat Release (HR) method (another version of the basket heating technique well published in the literature) may not be correct, especially for those measured at elevated oven temperatures and for larger basket sizes. Thirdly, simulations were performed to illustrate that the CPT phenomenon does not just occur at the center of the basket but also occurs everywhere else in the sample. This can become a significant advantage for further development of the CPT method in terms of reducing experimental duration and improving reproducibility.
\end{abstract}

\section{Introduction}

Stockpiles or layers of certain organic materials, under undesirable conditions, can generate sufficient heat to cause self-heating and then perhaps ignition. A typical

\footnotetext{
'Department of Chemical and Materials Engineering The University of Auckland, Private Bag 92019. Auckland, New Zealand. Email: d.chen@auckland.ac.nz

(C) Australian Mathematical Society 2001, Serial-fee code 0334-2700/01
} 
example is that, during spray drying of milk, layers of powder tend to form at various surfaces inside the spray dryer and other powder handling devices $[2,14]$. Some milk powder layers have been found to become a source of ignition.

Much work has been carried out on the self-heating of reactive solid materials theoretically and experimentally. For the self-heating of coal, in particular, there has been a constant stream of publications throughout the twentieth century [1]. Although the coals as mined always contain water, many theoretical studies only deal with 'dry coals' $[4,5,25]$. A series of previous studies on the self-heating of coal has been summarised by Carras and Young [6]. The increasing importance of the effect of water content on the self-heating of moist solids has been recognized and a series of related theoretical work has been conducted recently [24, 31, 32, 36, 37]. The mathematical models published, however, have been explored without quantitative comparison with experimental results. The relationship, as applied in these studies, between air humidity and water content of the porous solids, for instance, is not so realistic [8]. This paper firstly describes a fundamental mathematical model, developed based on our own recent studies $[8,10,17]$, which couples the effects of heat transfer, mass transfer and exothermic reactions. The model has been solved using an explicit computational scheme, which yields results that are compatible with recent laboratory results on temperature development in the basket heating of several milk powders [10]. Numerical investigations have been carried out, in this study, to help understand better the dynamics of the basket-heating system. In particular, the cross over behaviour and the effects of high humidity and high initial water content have been studied. Because the associated computer program implementing the model has been developed aiming at practical design for a safety practice that involves specific materials, no attempt has been made to normalize the variables to those commonly used for general bifurcation analysis.

\subsection{The 'cross over' behaviour and the crossing-point temperature method for} measuring activation energy and exothermicity In this paper, the model study of the cross over behaviour is described. This behaviour was first described by Gray $e t$ al. $[22,23]$. It was shown that self-heating occurs at the periphery of a sample before self-heating begins at its centre. In 1995, Chen and Chong [12] exploited the above behaviour experimentally and numerically and pointed out that such a behaviour can be used in kinetics measurement. In our laboratory, Chong et al. [18-20] carried out successful measurements on various milk powders and wood sawdusts using such a technique and considered some of the technical issues summarised by Chen and Chong [13]. They examined the behaviour of wood sawdust (fresh and chemically treated) and several types of milk powder. This approach was later adopted by other researchers in Australia [38], the US [21] and the UK [33] on a wider range of materials.

To illustrate the principle of the CPT method for obtaining the kinetic data, the 
energy conservation equation for a porous reactive infinite solid slab, neglecting reactant depletion and evaporation, is used for simplicity:

$$
\rho C_{p} \frac{\partial T}{\partial t}=\frac{\partial}{\partial x}\left(k \frac{\partial T}{\partial x}\right)+Q \rho A \exp (-E / R T),
$$

where the term on the left-hand side of the equation is the local rate of enthalpy change in the solid, the first term on the right-hand side is the conductive heat transfer in the solid and the second term on the right-hand side is the heat generation term of one exothermic reaction (or several reactions occurring simultaneously but considered to be one overall reaction).

If the solid has a uniform temperature to start with and this initial temperature is lower than the ambient temperature used in heating, then at some point during heating the heat conduction term in the symmetry of the slab is zero. At this point, (1) becomes

$$
\left.\frac{\partial T}{\partial t}\right|_{T=T_{p}}=\frac{Q A}{C_{p}} \exp \left(-E / R T_{p}\right)
$$

or, when rearranged,

$$
\left.\ln \frac{\partial T}{\partial t}\right|_{T=T_{p}}=\ln \frac{Q A}{C_{p}}-\frac{E}{R T_{p}} .
$$

The temperature at which the conduction term is zero has been defined as the 'crossingpoint temperature', $T_{p}[12]$. By measuring $T_{p}$ and its corresponding rate of change in temperature at the centre, a plot of $\ln (\partial T / \partial t)$ against the reciprocal of $T_{p}$ produces a straight line (or sections of straight lines or a continuous curve) with a slope of $-E / R$ and an intercept of $\ln \left(Q A / C_{p}\right)$. From the kinetic plot, and knowing the specific heat, $C_{p}$, of the powder, $E$ and $Q A$ (the product of the heat of reaction and the reaction frequency factor) are determined. Slight modification of the data analysis is needed if the specific heat capacity is temperature dependent [13]. It is possible to determine $T_{p}$ by measuring the temperature of two points in a sample: one at the centre, and the second at a point near the centre, on the same horizontal plane provided the symmetry of the sample is perpendicular to the horizon. This can be done by heating a cubic steel mesh basket or an equi-cylinder steel mesh basket or some other symmetrical basket in a temperature-controlled laboratory oven with air circulation [13]. One can also use a long cylinder (large length to diameter ratio) basket so that the end effect can then be neglected.

A standard testing procedure may be summarized as follows:

First of all, solid particles are packed into a steel mesh basket which has a steel mesh cover. The basket is typically of a few centimetres in diameter or side length. The basket containing the sample is then placed in the preheated oven (before the oven reaches its final temperature). Two thermocouples are inserted into the sample through 

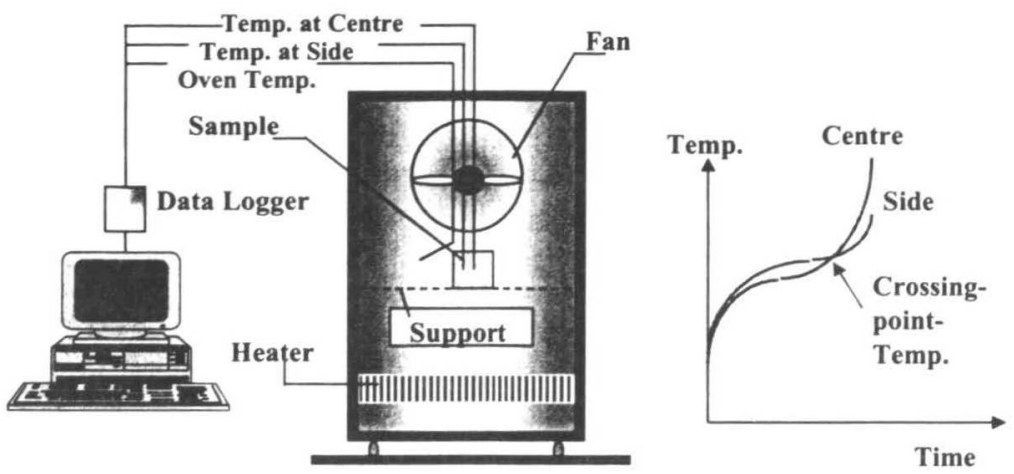

FIGURE 1. Schematic diagram of the basket-heating device and qualitative temperature records (showing the crossing point measurement).

guiding holes in the basket lid, one at the centre and the other several millimetres (this usually means 'several particle diameters') from the centre, on the same horizontal plane. Samples of different sizes for milk powder have been tested this way and the kinetics obtained were essentially the same $[18,20]$. They are connected to a data acquisition system which is connected to a computer (refer to Figure 1). A third thermocouple, also connected to the data logger, is set up to measure the oven temperature. The oven temperatures used are generally greater than $140^{\circ} \mathrm{C}$ as lower temperatures do not cause sufficient heating for the sample sizes used. The data acquisition program is set up such that the two thermocouples in the sample measure the centre temperature and the difference in temperature between the two points. The test is run until the difference in temperature crosses zero (when the centre temperature is higher than the temperature of the other point in the sample). The crossing-point temperatures are determined from the temperature-time curves.

The CPT method [13] has a number of advantages over the traditional basket heating method [3]:

- it only uses one size basket and thus is less restricted by the oven size available;

- it does not require an infinite Biot number boundary condition so the fan power need not be too great;

- it needs only one experimental run to determine one point on the kinetic plot;

- it can establish the effect of temperature on kinetic parameters (because values $Q A$ and $E$ are measured at the temperature at which the reaction occurs);

- this method can also be used to investigate a process without gaseous reactions (that is, gas-less reactions).

As such, the CPT method is very cost-effective compared to the more traditional basket heating method where steady-state Frank-Kamenetskii theory is used. The mathematical model described in this paper is a generic model. It is expected to be applicable for other particulate materials. 


\section{The mathematical model}

2.1. Governing equations for energy and mass conservation The moisture movement within the domain involves the phase change (evaporation/condensation) and transfer of moisture from the solid particles to the voids in-between. Water vapour is transferred through the voids towards the outer boundary of the domain of packed particulate and is dispersed into the ambient air by convection. Heat transfer is coupled with the phase change and movement, and is also through the conduction mechanism within the packed mass and by convection around the outer boundary of the packed particulate domain [17]. An illustration of the system is given in Figure 2.

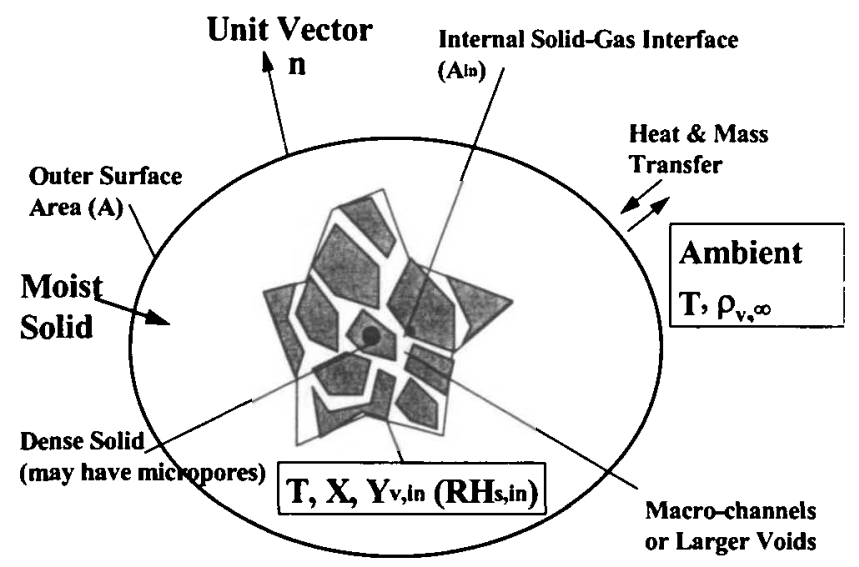

FIGURE 2. A schematic diagram of the transport processes in packed solid particles.

The following assumptions are made for the model to be described:

- negligible reactant consumption (oxygen, solid reactants);

- no convection inside the powder mass;

- Newtonian cooling at the boundary;

- constant thermal conductivity and diffusion coefficient of water vapour in the air;

- no temperature difference between the solid particles, moisture and gas in the voids;

- negligible (liquid) moisture transfer through the solid contacts, and the moisture transfer from the inner solid mass to the outer boundary (the outlining boundary of the entire particulate mass) is essentially by diffusion of water vapour.

These or similar assumptions (except the first which is related to chemical reaction engineering) are commonly used in heat/mass transfer in packed beds. The first 
assumption is based on the proof given by Bowes [3] for reactants with large activation energies.

Energy conservation Based on the above description, the governing equation for conservation of energy in a domain of packed exothermically reactive solid particles, taking into account drying/wetting, is written as:

$$
\rho C_{p} \frac{\partial T}{\partial t}=k \nabla \cdot \nabla T+Q \rho_{s d} A \exp \left(-\frac{E}{R T}\right)+\rho_{s d} H_{v} \frac{\partial X}{\partial x},
$$

where I is the local rate of enthalpy change in the solid, II is the conductive heat transfer in the solid, III is the heat generation term of the lumped exothermic reactions, and IV is the rate of heat release (or gain) due to drying (or wetting); $H_{v}$ is the heat of drying/wetting $\left(\mathrm{J} \mathrm{kg}^{-1}\right)$ and $X$ is the moisture content of the solid on a dry basis ( $\mathrm{kg} \mathrm{H} \mathrm{H}_{2} \mathrm{O} / \mathrm{kg}$ solid). In this work, $\rho C_{p}$ is a bulk solid property which is influenced by both the dry solids and porosity. In the current approach, $k$ has been assumed to be constant for each solid sample used in the laboratory work. This is simply because of the non-existence of the data for elevated temperatures. The particles considered have very low moisture contents (that is, a few percent). In this study, (1) has been applied to the packed dairy powders in a stainless steel basket of a regular shape.

The second term in (1) can be replaced, for regular geometry (like a slab, cylinder, sphere or cube), by $k \nabla \cdot \nabla T=k\left(\partial^{2} T / \partial x^{2}+(j / x) \partial T / \partial x\right)$ [3]. This replacement is used in this study. We define $j$ to be the shape factor (for example, $j=0$ for a slab, 1 for an infinite cylinder, 2 for a sphere, and 3.28 for a cube) (dimensionless). In this study, equi-cylinder baskets were used for experimental validation. The $j$ value was taken to be 2.728 [3].

Mass conservation For liquid water in solid particles, the transfer of moisture from solid particles into the surrounding voids is given by

$$
-\rho_{s d} \frac{\partial X}{\partial t}=h_{m, i n} A_{p} n_{p}\left(Y_{s, i n}-Y\right),
$$

where $h_{m, i n}$ is the effective mass transfer coefficient between the particle and gas in the voids $\left(\mathrm{m} \mathrm{s}^{-1}\right), A_{p}$ is the surface area of one particle $\left(\mathrm{m}^{2}\right), n_{p}$ is the number of particles per unit volume $\left(1 \mathrm{~m}^{-3}\right)$ (that is, the particle concentration), $Y_{s, \text { in }}$ is the vapour concentration at the surface of the particle $\left(\mathrm{kg} \mathrm{m}^{-3}\right)$ and $Y$ is the vapour concentration in the voids of the bulk solid $\left(\mathrm{kg} \mathrm{m}^{-3}\right)$. Equation (4) is a simplified model because the effect of internal water transfer (diffusion of water at very low water content) is not explicitly expressed (otherwise, one needs to solve another partial differential equation for each particle). Also $h_{m . i n}$ is essentially an overall mass transfer coefficient through a composite of a solid and an air layer. 
One can define $Y_{s, i n}$ using the following relationship for the relative humidity $R H_{s}$ at the surface of the particle:

$$
R H_{s}=\frac{Y_{s, i n}}{Y_{s a t}}
$$

where $Y_{s, i n}$ is the partial vapor density at the surface of the particle (Pa) and $Y_{s a t}$ is the vapour density at saturation $(\mathrm{Pa})$.

The product of the surface area of one particle and the particle concentration in (2) (that is, $A_{p} n_{p}$ ) was calculated using the volume and the bulk density of the samples used in laboratory tests. The overall value of $A_{p} n_{p}$ was taken to be the sum of the contributions from the particles in each size fraction [17]. The term $Y_{s a t}(T)$ is a function of temperature, and may be represented by

$$
Y_{s a t}(T)=K_{\nu} \exp (-E / R T),
$$

where $K_{v}$ is the apparent 'reaction frequency' for evaporation $\left(\mathrm{kg} \mathrm{m}^{-3}\right)$ and $E_{v}$ is the activation energy for pure water evaporation $\left(\mathrm{J} \mathrm{mol}^{-1}\right)$; ' $v$ ' denotes vapour [15]. A correlation for $Y_{\text {sat }}(T)$ at atmospheric conditions for temperatures between 0 and $100^{\circ} \mathrm{C}$ gave the values of $K_{v}$ and $E_{v}$ as $2.62 \times 10^{5} \mathrm{~kg} \mathrm{~m}^{-3}$ and $40.21 \mathrm{~kJ} \mathrm{~mol}^{-1}$ respectively. By substituting the correlation into the local mass transfer equation, the following equation is obtained for liquid water conservation in the particles:

$$
-\frac{\partial X}{\partial t}=\frac{h_{m} A_{p} n_{p}}{\rho_{s d}}\left[R H_{s} K_{v} \exp \left(-E_{v} / R T\right)-Y\right] .
$$

To determine the overall rate of phase change in the bulk solid, the particle surface area to volume ratio in (7) was multiplied by the number of particles. The particle surface relative humidity (inside the powder bed, when near equilibrium) can be correlated in the following form using a reaction engineering approach proposed by Chen $[9,11]$ :

$$
R H_{s}=\exp \left(-E_{w} / R T\right) \text { and } E_{w}=a X^{-b} / T^{n-1},
$$

where $a, b, n$ are positive constants, and $E_{w}$ is an apparent activation energy $\left(\mathrm{J} \mathrm{mol}^{-1}\right)$. The temperature dependence of the apparent activation energy reflects the change in the interactions between water and the foods at different temperatures. The value of $n$ reflects how strongly such a relationship depends on temperature and can vary from 0 to 25 for a wide range of food materials tested [11]. The correlation equations for whole and skim milk powders were derived using data from the literature [29, 35].

For water vapour in voids in-between particles, the mass balance equation for the water vapour in the bulk, $Y$, is given by:

$$
\frac{\partial Y}{\partial t}=\varepsilon D\left(\frac{\partial^{2} Y}{\partial x^{2}}+\frac{j}{x} \frac{\partial Y}{\partial x}\right)-\rho_{s d} \frac{\partial X}{\partial t}
$$


where $\varepsilon$ is the porosity of the packed powder bed and $D$ is the diffusion coefficient for water in air $\left(\mathrm{m}^{2} \mathrm{~s}^{-1}\right)$, which is assumed to be constant throughout the mass. The porosity here represents the void fraction in between the particles and is determined using the bulk density and the particle density of the material of concern.

2.2. Boundary conditions The general boundary conditions for (3) are

$$
\frac{\partial T}{\partial t}=\frac{k(j+1)}{\rho C_{p}} \frac{\partial^{2} T}{\partial x^{2}}+\frac{Q \rho_{s d} A}{\rho C_{p}} e^{-E / R T}+\frac{\rho_{s d} H_{v}}{\rho C_{p}} \frac{\partial X}{\partial t} \quad \text { at } x=0
$$

and

$$
-k \frac{d T}{d x}=h\left(T-T_{\infty}\right) \quad \text { at } x=r,
$$

where $h$ is the heat transfer coefficient at the interface between the bulk solid and ambient air $\left(\mathrm{W} \mathrm{m}^{-2} \mathrm{~K}^{-1}\right)$ and $T_{\infty}$ is the ambient temperature $(\mathrm{K})$. Here $r$ is the basket half-width (m).

For mass transfer, the boundary conditions for the vapour concentration equation are

and

$$
\frac{\partial Y}{\partial t}=\varepsilon D(j+1) \frac{\partial^{2} Y}{\partial x^{2}}-\rho_{s d} \frac{\partial X}{\partial t} \quad \text { at } x=0
$$

$$
-\varepsilon D \frac{d Y}{d x}=h_{m, o u t}\left(\rho_{v, s}-\rho_{v, \infty}\right) \quad \text { at } x=r,
$$

where $h_{m . o u f}$ is the mass transfer coefficient between the outer surface of the powder mass and the ambient gas $\left(\mathrm{m} \mathrm{s}^{-1}\right)$, and $\rho_{v, \infty}$ is the ambient water vapour density $\left(\mathrm{kg} \mathrm{m}^{-3}\right)$; ' $s$ ' denotes the outer powder mass-gas interface and $\rho_{v, s}$ is correlated to $Y$ at $x=r$ by

$$
\rho_{v, s}=\left.\frac{Y}{\varepsilon}\right|_{x=r} .
$$

The heat and mass transfer coefficients in the test oven were determined using the data obtained from a series of water evaporation tests. For the conditions tested, the heat and mass transfer coefficients were correlated empirically to the oven temperature [17].

2.3. Physical properties The chemical and physical properties used in the simulations were largely taken from the literature and many of them are temperature dependent. The parameters of reaction kinetics used for the skim milk powder had been obtained experimentally [17-20] $\left(E=97.1 \mathrm{~kJ} \mathrm{~mol}^{-1}\right.$ and $\left.\ln \left(Q A / C_{p}\right)=22.3\right)$. The thermal conductivities of the skim milk powders were calculated from correlations in the literature [30]. The thermal conductivities ranged from 0.03 to $0.11 \mathrm{~W} \mathrm{~m}^{-1} \mathrm{~K}^{-1}$ 


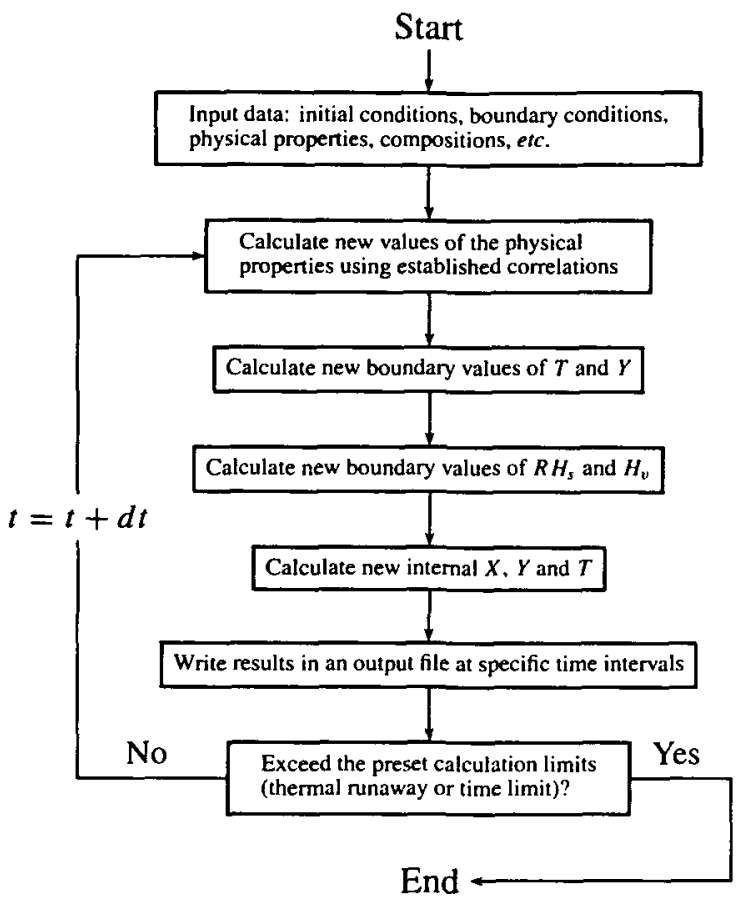

FIGURE 3. Flow chart of the current computer program.

for skim milk powder [30]. The specific heat capacity of the milk powder (as a sum of the fractions of the specific heat capacities of fat, protein, carbohydrate, moisture and ash) was estimated using the correlations given by Choi and Okos [16]. The specific heat capacity of the bulk milk powder was calculated by taking into account the air between particles. The bulk density for the skim milk powder was $700 \pm 5 \mathrm{~kg} \mathrm{~m}^{-3}$ [17]. The diffusion coefficient of water vapour in the air was required and was assumed constant at $2.6 \times 10^{-5} \mathrm{~m}^{2} \mathrm{~s}^{-1}$ [26]; $h_{m}$ was found to be $8 \times 10^{-7} \mathrm{~m} \mathrm{~s}^{-1}$ [17].

The equations for internal surface relative humidity were correlated using data obtained from the literature. For skim milk powder the equilibrium sorption isotherm is expressed as

$$
R H_{s}=\exp \left(-\frac{0.003106}{R}\left(\frac{1}{X^{2.18}}\right)\left(\frac{308}{T}\right)^{15}\right) .
$$

The heat of drying/wetting, $H_{v}$, is the heat required to remove water from the solids or the heat released when wetting occurs. It includes the latent heat and the additional heat required to remove the small remaining moisture in the solids. Because of the empirical nature of (15), $R H$ becomes an extremely small value as $X$ approaches 
zero. A compromise has been made to set $X$ to zero as soon as $R H$ reaches $1 \times 10^{-4}$. In this study, the modified version of an approximate equation was used according to Chen [10]:

$$
H_{v}=L_{v}-f \frac{R T}{M_{\mathrm{H}_{2} \mathrm{O}}} \ln \left(R H_{s}\right),
$$

where $L v$ is the latent heat of vaporisation of water $\left(\mathrm{J} \mathrm{kg}^{-1}\right)$ and $M_{\mathrm{H}_{2} \mathrm{O}}$ is the molecular mass of water $\left(\mathrm{g} \mathrm{mol}^{-1}\right) ; f$ is taken as $\sim 10$ (which is in the practical range for milk powders). This equation is consistent with that derived from the Clausius-Clapeyron equation in conjunction with the empirical correlation for $R H$ vs. $X$ at different $T$ [34]. A preliminary sensitivity analysis of the parameter space has been tested in [17] and thus it is not the aim in this study. Note that the physical properties involved in the model all have good physical backgrounds and the order of magnitude for each parameter has been reliably estimated for the milk powder of concern.

2.4. The numerical method The numerical scheme is straightforward, that is, it is the explicit finite difference method which can be found in the general heat transfer literature (for example, see [26]). For instance, the energy balance is approximated by:

$$
\begin{aligned}
\rho C_{p}\left(T_{i}^{m-1}\right) & \frac{T_{i}^{m}-T_{i}^{m-1}}{\Delta t} \\
\approx & k\left(\frac{T_{i+1}^{m-1}-2 T_{i}^{m-1}+T_{i-1}^{m-1}}{\Delta x^{2}}+\frac{j}{x_{i}} \frac{T_{i+1}^{m-1}-T_{i}^{m-1}}{\Delta x}\right)+Q \rho_{s d} A e^{-E / R T_{i}^{m-1}} \\
& +\rho_{s d} H_{v}\left(T_{i}^{m-1}, R H_{i}^{m-1}\right)\left(\frac{\Delta X}{\Delta t}\right)_{i}^{m},
\end{aligned}
$$

where $\Delta x$ is the distance increment, $\Delta t$ is the time increment, $m-1$ represents the previous timing, $m$ represents the current timing (that is, $t^{m}=t^{m-1}+\Delta t$ ), $i$ labels the geometric location of the node of concern and, $i-1$ and $i+1$ are the nodes which are immediately next to node $i$ within the sample domain. The present code has been named "Diffusive Self-Heating Code I (DSCI)".

The program is written in Fortran 77. The customized block diagram of the program is given in Figure 3. Uniform grid size is used for simplicity (commonly 25, 50 grids are used for a sample size of a few centimetres). For simplicity, a quasi-steady state, such that $\partial Y / \partial t \approx 0$, was assumed (implying that the moisture transfer from the solid particles to the voids is the limiting process). The computer program was set up to solve for $X$, then $Y$ and $T$ for each node before repeating the procedure for the next time step. The number of nodes was 25 , and the time step was $0.01 \mathrm{~s}$ (little difference was found for finer meshes). 


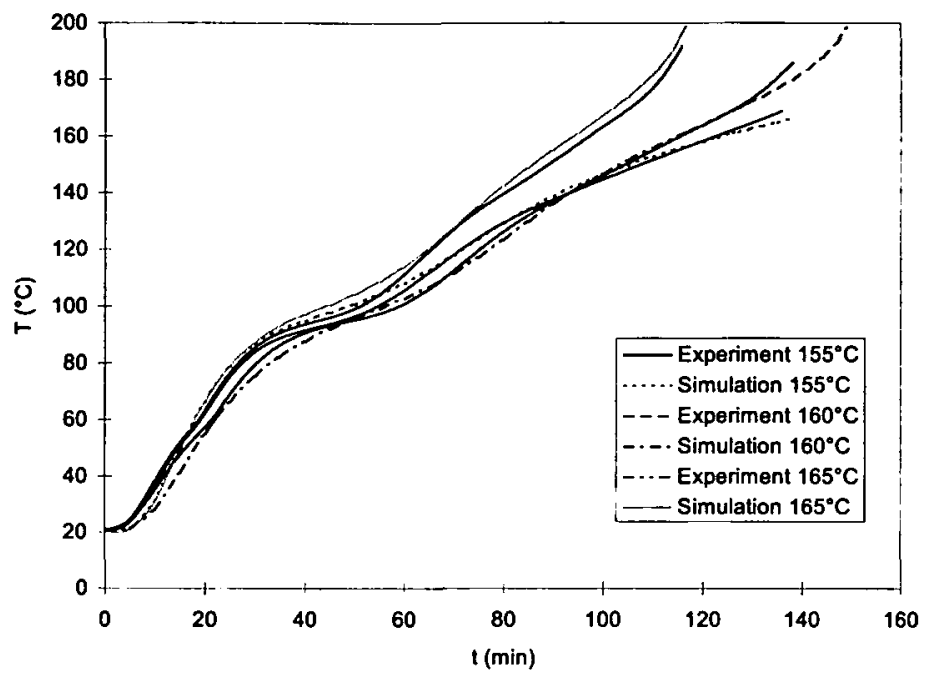

FiguRE 4. A comparison between the experimental and predicted temperature-time profiles for the skim milk powder contained in an equi-cylinder basket.

\section{Results and discussion}

3.1. Comparison with experimental results Firstly, we show that the model with appropriate physical properties and reaction kinetics can predict well the measured results (in particular, good agreements have been gained for the lower temperature range). In these experiments, a standard laboratory oven of $50 \times 55 \times 55 \mathrm{~cm}$ was used. A standard skim milk powder was used, which has a water content of $0.04 \mathrm{~g} / \mathrm{g}$ on a dry basis. Each milk powder sample was placed and weighed in a no. 30 stainless steel mesh basket, which was then placed at the centre of the temperature-controlled oven. Air circulation in the oven was provided by an inbuilt fan. The initial sample temperature was $27^{\circ} \mathrm{C}$ and the relative humidity at this temperature was $50 \%$. Stainless steel mesh baskets used were of equi-cylinder type (with a radius being $2.5 \mathrm{~cm}$ ). Two Type $\mathrm{K}, 1 \mathrm{~mm}$ in diameter, thermocouples were inserted in the same horizontal and vertical cross-sectional plane. The first thermocouple was fixed at the geometric centre of the sample. The distance between the two thermocouples was $6 \mathrm{~mm}$. In each experiment, the temperature-time profile during heating was recorded using a data acquisition system. A typical comparison is given in Figure 4. The simulation predicted the water evaporation region quite accurately. In general, the predicted selfheating behaviour is fairly comparable to the behaviour observed in the experiments (for a low temperature range in particular). Thermal conductivity was found to have an effect on the temperature rise in the initial stage of the process [17]. Unfortunately, at this stage, we do not have accurate temperature-dependent thermal conductivity available at high temperatures. 


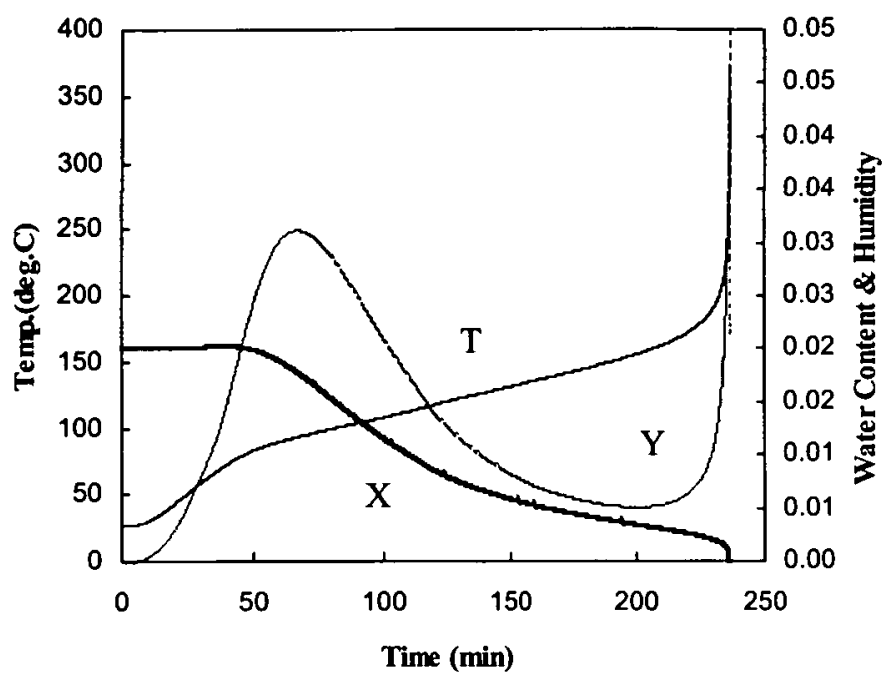

FIGURE 5. Predicted temperature/moisture content/humidity changes with time $\left(R H_{\infty}=10 \%\right.$ at $\left.27^{\circ} \mathrm{C}\right)$. This curves are identical to the ones predicted with $100 \%$ ambient $R H$ at the same room temperature (totally overlapped).

Comparison of the model predictions with the actual experimental data has allowed us to study further the behaviour and impact of the cross over behaviour with a view to improving the CPT measuring technique as mentioned in the introduction. The following sections will describe our new insights about this phenomena.

3.2. The influence of ambient humidity upon the self-heating process We set up a program to incorporate the effect of ambient relative humidity on the self-heating process in laboratory tests. The ambient relative humidity at room temperature (which was also the initial temperature of the milk powder sample) was specified in the input data file [17]. This ambient air would be drawn into the oven during each test due to the air circulation mechanism of the laboratory oven. The case simulated here involves an infinite slab of packed skim milk powder and $r=2 \mathrm{~cm}$. The initial water content is $0.02 \mathrm{~g} / \mathrm{g}$ on a dry basis. The oven temperature is $165^{\circ} \mathrm{C}$. Figure 5 shows the time responses predicted for a dry ambient with $10 \%$ relative humidity at ambient temperature. The ones with $100 \%$ relative humidity at room temperature are almost identical to the dry ones. This is because despite the large difference in room relative humidity, after the air is drawn into the oven controlled at $165^{\circ} \mathrm{C}$ the $R H$ in the oven is still very low. As such, under normal circumstances, ambient humidity fluctuations in the laboratory would not influence the heating profile much at $165^{\circ} \mathrm{C}$ or higher.

It is also interesting to note that, in the time responses of humidity shown in Figure 5, the humidity (vapour concentration) rises rapidly towards the end of the drying-out period of the liquid water content of the sample, and then drops rapidly as the powder 


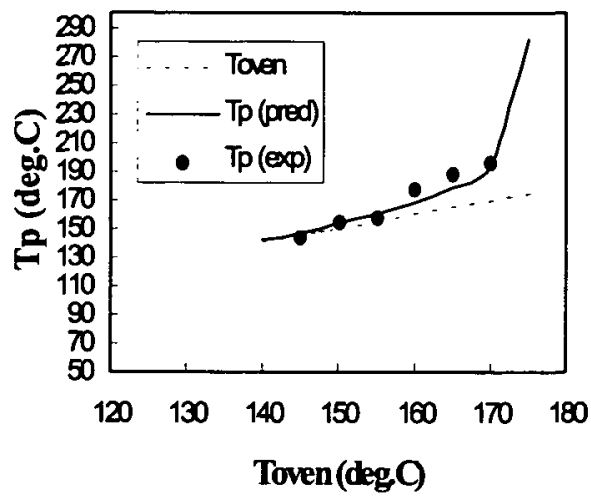

(a)

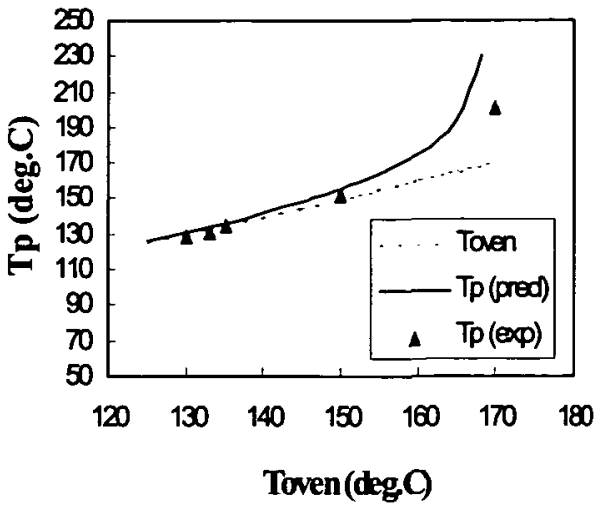

(b)

FIGURE 6. The departure of $T_{p}$ from $T_{o}$ (Toven) as predicted by the model and recorded experimentally on the skim milk powder for (a) the cubic basket size $2 r=5 \mathrm{~cm}$; (b) $2 r=6 \mathrm{~cm}$.

is dried completely. This is in good agreement with the previous predictions by Chen using a simpler model [10].

\subsection{Simulations carried out to further explore the 'cross over' behaviour As} mentioned in the introduction, there exists a 'cross over' behaviour (that is, the occurrence of the CPT) when heating up a symmetrical exothermically reactive solid which is initially at a uniform temperature that is lower than the ambient temperature. This behaviour is the fundamental reason for the success of the CPT method for measuring exothermic reactivity of the solid. As shown in Figure 5, for the skim milk powder (under the lab conditions specified earlier; cubic samples of $r=2.5$ and $3 \mathrm{~cm}$ were used respectively), the mathematical model predicts very well such a cross over behaviour which was observed experimentally. One key feature of these diagrams is that they show the clear departure of the crossing-point temperature away from the oven temperature as the oven temperature increases. This departure has a clear impact on the experimental approach taken by Jones et al. $[27,28]$ who applied the following equation to measure reactivity:

$$
q=C_{p} \rho(d T / d t)_{0}=Q \rho A e^{-E / R T_{0}},
$$

where $q$ is the total heat release. This approach is called the "Heat Release" (HR) method. Although it looks similar to (2) proposed by Chen and Chong [12,13], the difference is that $T_{0}$ here is the oven temperature rather than the crossing-point temperature $T_{p}$. Essentially the measurements of Jones et al. $[27,28]$ were taken using the above equation with the heat conduction term at the centre, when it reached the oven temperature, being neglected. Both the simulation and the experimental results 


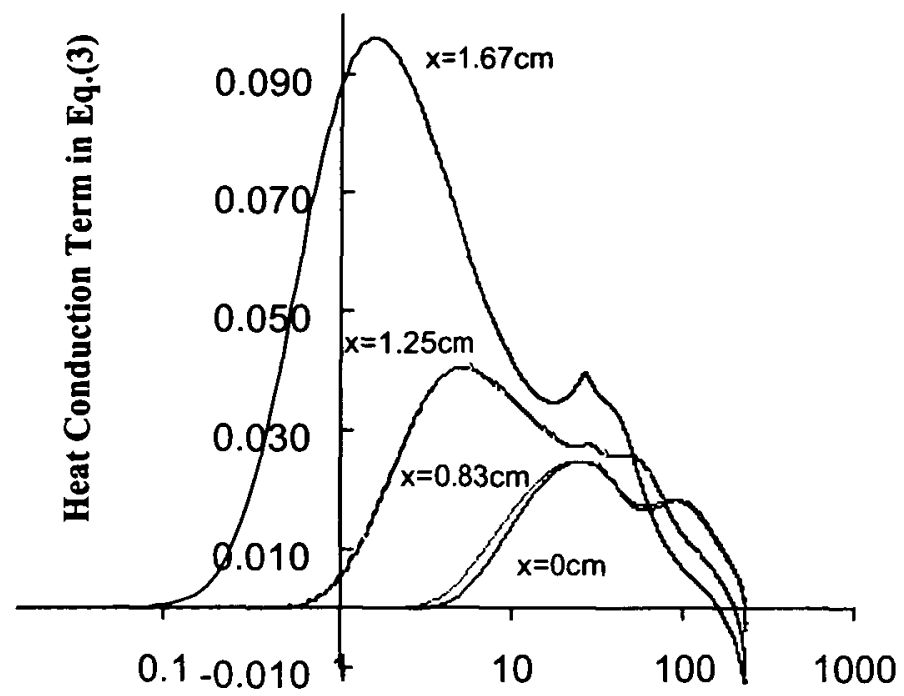

Time (min)

FIGURE 7. Evolution of the heat conduction term in (3) against heating time (at four locations, $x=0$ (that is, the centre), $x=0.83 \mathrm{~cm}, x=1.25 \mathrm{~cm}$ and $x=1.67 \mathrm{~cm}$ ).

of $T_{p}$ shown in Figure 6 suggest that the HR approach may lead to considerable error. The work carried out by the Leeds Group on coal has shown that the CPT method devised by Chen et al. [17-19,27,28] gave basically the same results as those by Frank-Kamenetskii theory. It is difficult to check, however, whether the HR method is valid under the circumstances tested as it ignores a fundamental aspect. This is expected to be due to the phenomena shown in Figure 6. Indeed, our experimental results of $T_{p}$ at various oven temperatures on wood particles [19] also indicate that the departure of $T_{p}$ from the corresponding $T_{0}$ is very significant as $T_{0}$ gets higher [7].

\subsection{Simulations carried out to show that the CPT can be found 'everywhere'} As described earlier, one can definitely identify a crossing-point at the centre of a reactive symmetrical body. Now, it can be shown that, in fact, this cross over behaviour also happens in the rest of the sample being tested in the CPT procedure. Using arguments very similar to those put forward by Gray et al. [22], one can prove that, in the same horizontal plane where the centre temperature is taken, at one point of time, each location would pass its own crossing-point. In other words, at this location (or point) $\partial^{2} T / \partial x^{2}=0$ (note here that a uniform $k$ value has been assumed). Figure 7 shows the profiles of $\partial^{2} T / \partial x^{2}=0$ vs. $x$ at different timings (the conditions simulated are the same as those in Figure 4) based on the current simulation results. This 'generalization' of the cross over behaviour may be used in future CPT method development. By inserting a few more thermocouples, each basket-heating run would 
yield several data points (instead of just one) on the kinetic plot for calculating the kinetic parameters.

\section{Conclusions}

A detailed mathematical model is described here and the predictions made using the model are compared with the available experimental data. The 'drawbacks' of the mathematical model are associated with the fact that in reality the physical properties of the materials need to be correlated using some 'non-ideal' formats. Improvement in precision of these semi-empirical equations is desired. In any case, the model is reliable for the practical purposes at present. The behaviour in laboratory tests has been simulated adequately. The model can also be used to explore more realistic situations in industry. The 'one-dimensional' model can be extended into two- and three-dimensional systems.

In this study, the cross over phenomenon has been further examined with the model and the predictions combined with the experimental evidence show that the reaction kinetics obtained using the Heat Release (HR) method (also a version of the basket heating technique) may not be correct. Furthermore, simulations have shown that the CPT phenomena can be a common feature within symmetrically heated bodies and this result can be taken into account for further development of the CPT method to reduce testing time and improve reproducibility.

\section{Acknowledgement}

This paper was submitted to the Combustion Meeting in honour of Prof. Brian Gray.

\section{References}

[1] S. C. Banerjee, Spontaneous Combustion of Coal and Mine Fires (A. A. Balkema, Rotterdam, India, 1985).

[2] P. F. Beever and D. Crowhurst, "Fire and explosion hazards associated with milk spray drying operations", J. Soc. Dairy Tech. 42 (1989) 65-70.

[3] P. C. Bowes, Self-heating: Evaluating and controlling the hazards (Elsevier, Amsterdam, The Netherlands, 1984).

[4] S. Bradshaw, D. Glasser and K. Brooks, "Self-ignition and convection patterns in an infinite coal layer”, Chem. Eng. Communications 105 (1991) 255-278.

[5] K. Brooks, N. Svanas and D. Glasser, "Evaluating the risk of spontaneous combustion in coal stockpiles", Fuel 67 (1988) 651-656.

[6] J. N. Carras and B. C. Young, "Self-heating of moist coal", Progress in Energy and Combustion Sci. 20 (1994) 1-15. 
[7] X. D. Chen, "Basket heating methods for obtaining exothermic reactivity of solid materiais. Part I. The extent and impact of the departure of the crossing-point temperature from the corresponding oven temperature", Trans.IChemE Part B, (submitted).

[8] X. D. Chen, "On the mathematical modelling of the transient spontaneous heating in a moist coal stockpile", Combustion and Flame 90 (1992) 114-120.

[9] X. D. Chen, "A new water sorption equilibrium isotherm model", Food Research Internat. 30 (1998) 755-759.

[10] X. D. Chen, "On the fundamentals of diffusive self-heating of water containing combustible materials", Chem. Eng. and Processing 37 (1998) 367-378.

[11] X. D. Chen, "Temperature dependence function of equilibrium sorption isotherms established by a reaction engineering approach", J. Food Engineering 37 (1998) 259-269.

[12] X. D. Chen and L. V. Chong, "Some characteristics of transient self-heating inside an exothermically reactive porous solid slab", Trans. IChemE 73B (1995) 101-107.

[13] X. D. Chen and L. V. Chong, "Several important issues related to the crossing-point temperature (CPT) method for measuring self-ignition kinetics of combustible solids", Trans. IChemE 76B (1998) 90-93.

[14] X. D. Chen, R. Lake and S. Jebson, "Study of milk powder deposition on a large industrial drier", Trans. IChemE 71C (1993) 180-186.

[15] X. D. Chen and G. Z. Xie, "Fingerprints of the drying behaviour of particulate or thin layer food materials established using a reaction engineering model", Trans. IChemE 75C (1997) 213-222.

[16] Y. Choi and M. R. Okos, "Effects of temperature and composition on the thermal properties of foods", in Food Engineering and Process Applications Volume 1: Transport Phenomena (eds. M. Le Maguer and P. Jelen), (Elsevier Applied Science Publishers, London, 1986) 93-101.

[17] L. V. Chong, "Thermal ignition of dairy powders", Ph. D. Thesis, Department of Chemical and Materials Engineering, The University of Auckland, Auckland, New Zealand, 1997.

[18] L. V. Chong, X. D. Chen and A. R. Mackereth, "Effect of composition on the thermal ignition kinetics of milk powders", in Proceedings of the 25th Australasian Chemical Engineering Conference (Chemeca 97), on CD-ROM, 1997.

[19] L. V. Chong, I. R. Shaw and X. D. Chen, "Thermal ignition kinetics of wood sawdust measured by a newly devised experimental technique", Process Safety Progress 14 (1995) 266-270.

[20] L. V. Chong, I. R. Shaw and X. D. Chen, "Exothermic reactivities of skim and whole milk powders as measured using a novel procedure", J. Food Engineering 30 (1996) 185-196.

[21] B. R. Cuzzillo, "Pyrophoria", Ph. D. Thesis, Department of Mechanical Engineering, University of California at Berkeley, USA, 1997.

[22] B. F. Gray, S. G. Little and G. C. Wake, "The prediction of a practical lower bound for ignition delay times and a method of scaling times-to-ignition in large reactant masses from laboratory data - II", in 24th Symposium (Int.) on Combustion, (The Combustion Institute, 1992), 1785-1791.

[23] B. F. Gray, J. H. Merkin and J. F. Griffiths, "The prediction of a practical lower bound for ignition delay times and a method of scaling times-to-ignition in large reactant masses from laboratory data", in 23rd Symposium (Int.) on Combustion, (The Combustion Institute, 1990), 1775-1782.

[24] B. F. Gray and G. C. Wake, "The ignition of hygroscopic materials by water", Combustion and Flame 79 (1990) 2-6.

[25] A. S. Hull, J. L. Lanthier, Z. Chen and P. K. Agarwal, “The role of diffusion of oxygen and radiation on the spontaneous combustibility of a coal pile in confined storage", Combustion and Flame 110 (1997) 479-493.

[26] F. P. Incropera and D. P. De Witt. Fundamentals of Heat and Mass Transfer, 3rd ed. (John Wiley and Sons, New York, 1990).

[27] J. C. Jones, “On the thermal ignition of wood waste", Trans. IChemE 76 (1998) 205-210.

[28] J. C. Jones, P. S. Chiz, R. Koh and J. Matthew, "Continuity of kinetics between sub- and supercritical regimes in the oxidation of a high-volatile solid substrate", Fuel 75 (1996) 1733-1736. 
[29] K. Jouppila and Y. H. Roos, "Water sorption and time-dependent phenomena of milk powders", J. Dairy Sci. 77 (1994) 1798-1808.

[30] D. A. MacCarthy, "Effect of temperature and bulk density on thermal conductivity of spray-dried whole milk powder", J. Food Engineering 4 (1985) 249-263.

[31] A. C. McIntosh, "A Semenov approach to the modelling of the thermal runaway of damp combustible material", IMA J. Appl. Math. 51 (1993) 217-237.

[32] A. C. McIntosh, B. F. Gray and G. C. Wake, "The ignition of combustible material in the presence of a damp atmosphere", Phys. Lett. A 191 (1994) 61-71.

[33] Y. S. Nugrono, A. C. McIntosh and B. M. Gibbs, "Using the crossing-point temperature method to assess self-heating behaviour of Indonesian coals", in Proceedings of the 27th Int. Sym. on Combustion, University of Colorado at Boulder, Colorado, Aug. 3-7 (1998), 1998).

[34] S. Rahman, Food Properties Handbook (CRC Press, Boca Raton, Florida, 1995).

[35] Y. Sano and R. B. Keey, "The drying of a spherical particle containing colloidal material into a hollow sphere", Chem. Engineering Sci. 37 (1982) 881-889.

[36] R. A. Sisson, S. Swift, G. C. Wake and B. F. Gray, "The self-heating of damp cellulosic materials: I. High thermal conductivity and diffusivity", IMA J. Appl. Math. 49 (1992) 273-279.

[37] R. A. Sisson, S. Swift, G. C. Wake and B. F. Gray, "The self-heating of damp cellulosic materials: II. On the steady state of the spatially distributed case", IMA J. Appl. Math. 50 (1993) 285-306.

[38] D. K. Zhang, W. Sujanti and X. D. Chen, "A low-temperature oxidation study using wire-mesh reactors with both steady-state and transient methods", Combustion and Flame (1998), (in press). 